\title{
INTERIOR RECONSTRUCTION USING THE 3D HOUGH TRANSFORM
}

\author{
R.-C. Dumitru, D. Borrmann, and A. Nüchter
}

Automation Group, School of Engineering and Science

Jacobs University Bremen, Germany

andreas@nuechti.de

Commission WG V/4

KEY WORDS: interior reconstruction, 3D modeling, plane detection, opening classification, occlusion reconstruction

\begin{abstract}
:
Laser scanners are often used to create accurate 3D models of buildings for civil engineering purposes, but the process of manually vectorizing a 3D point cloud is time consuming and error-prone (Adan and Huber, 2011). Therefore, the need to characterize and quantify complex environments in an automatic fashion arises, posing challenges for data analysis. This paper presents a system for 3D modeling by detecting planes in 3D point clouds, based on which the scene is reconstructed at a high architectural level through removing automatically clutter and foreground data. The implemented software detects openings, such as windows and doors and completes the 3D model by inpainting.
\end{abstract}

\section{INTRODUCTION}

Recently, 3D point cloud processing has become popular, e.g., in the context of 3D object recognition by the researchers at Willow Garage and Open Perception using their famous robot, the PR2, and their Point Cloud Library (Willow Garage, 2012) for 3D scan or Kinect-like data. This paper proposes an automatic analysis of $3 \mathrm{D}$ point cloud data on a larger scale. It focuses on interior reconstruction from 3D point cloud data. The main tool of achieving this goal will be the 3D Hough transform aiding in the efficient extraction of planes. The proposed software is added to the $3 D T K$ - The 3D Toolkit (Lingemann and Nüchter, 2012) which already contains efficient variants of the 3D Hough transform for plane extraction (Borrmann et al., 2011). Furthermore, Adan and Huber (2011) state that there have been attempts to automate interior and exterior reconstruction, but most of these attempts have tried to create realistic looking models, instead of trying to achieve a geometrically accurate one. Now that 3D point cloud acquisition technology has progressed, researchers apply it to realistically scaled real-world environments. This research proposes to extend the work of Borrmann et al. (2011) and that of Adan et al. (2011); Adan and Huber (2011) by combining the approach of plane extraction and the approach of $3 \mathrm{D}$ reconstruction of interiors under occlusion and clutter, to automatically reconstruct a scene at a high architectural level.

Throughout the paper, we will demonstrate the algorithms using 3D data acquired with a Riegl VZ-400 3D laser scanner in a basement room (cf. Figure 1). After presenting related work, we describe our fast plane detection which is extended to reliably model walls. Afterwards, we describe our implementations of occlusion labeling and opening detection before we finally reconstruct the occluded part of the point cloud using inpainting.

\section{RELATED WORK}

There is a large body of research done in this field, mainly focusing on creating an aesthetically accurate model, rather than a geometrically accurate one (Adan and Huber, 2011). Papers that approach the issue from such a point of view are Früh et al. (2005); Hähnel et al. (2003); Stamos et al. (2006); Thrun et al. (2004).
Since architectural shapes of environments follow standard conventions arising from tradition or utility (Fisher, 2002) one can exploit knowledge for reconstruction of indoor environments. An interesting approach is presented in Budroni and Böhm (2005) where sweeping planes are used to extract initial planes and a geometric model is computed by intersecting these planes. In outdoor scenarios precise facade reconstruction is popular including an interpretation step using grammars (Böhm, 2008; Böhm et al., 2007; Pu and Vosselman, 2009; Ripperda and Brenner, 2009).

In previous work we have focused on interpreting 3D plane models using a semantic net and on correcting sensor data of a custommade 3D scanner for mobile robots (Nüchter et al., 2003). In this paper, we focus on precisely reconstructing a "room" model from a 3D geometric perspective.

\section{PLANE DETECTION}

The basis of the plane detection algorithm is the patent published by Paul Hough in 1962 (Hough, 1962). The common representation for a plane is the signed distance $\rho$ to the origin of the coordinate system, and the slopes $m_{x}$ and $m_{y}$ in direction of the $x$ - and $y$-axis. All in all, we parametrize a plane as $z=$

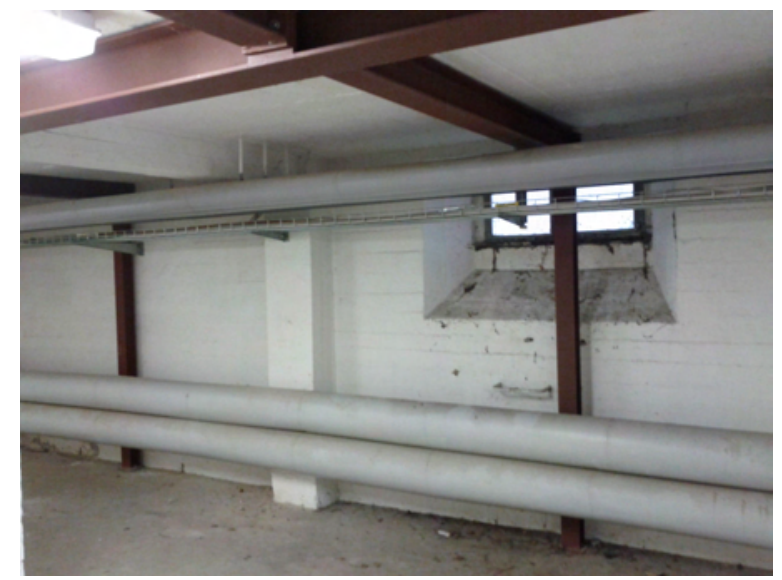

Figure 1: Example environment in a basement used throughout this paper. 

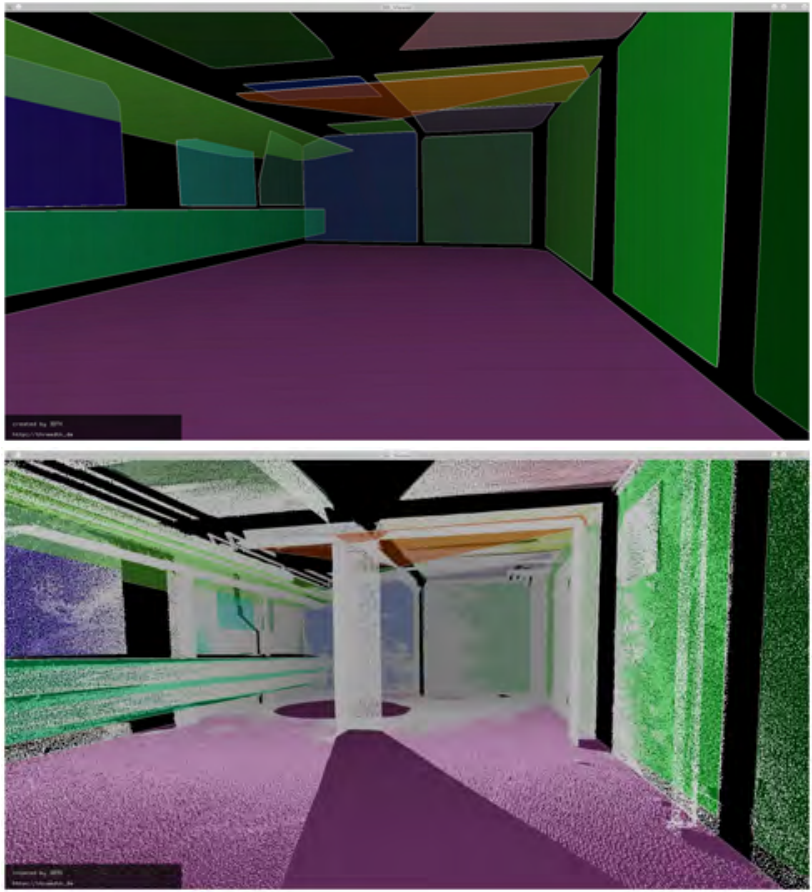

Figure 2: Plane detection in the fragmented basement room. Top: Detected planes. Bottom: Planes and 3D points.

$m_{x} \cdot x+m_{y} \cdot y+\rho$. To avoid problems caused by infinite slopes during the representation of vertical planes, the Hesse Normal Form is used, a method based on normal vectors. The plane is thereby given by a point $\mathbf{p}$ on the plane, the normal vector $\mathbf{n}$ that is perpendicular to the plane, and the distance $\rho$ to the origin. Therefore, the parametrization of the plane becomes:

$$
\rho=\mathbf{p} \cdot \mathbf{n}=p_{x} n_{x}+p_{y} n_{y}+p_{z} n_{z} .
$$

Considering the angles between the normal vector and the coordinate system, the coordinates of $\mathbf{n}$ are factorized to

$$
p_{x} \cdot \cos \theta \cdot \sin \varphi+p_{y} \cdot \sin \varphi \cdot \sin \theta+p_{z} \cdot \cos \varphi=\rho,
$$

with $\theta$ the angle of the normal vector on the $x y$-plane and $\varphi$ the angle between the $x y$-plane and the normal vector in $z$ direction. To find planes in a point set, we transform each point into the Hough Space $(\theta, \varphi, \rho)$. Given a point $\mathbf{p}$ in Cartesian coordinates, we must determine all the planes the point lies on. Marking these planes in the Hough Space will lead to a 3D sinusoid curve. The intersections of two curves in Hough Space denote the set of all possible planes rotated around the lines defined by two points. Consequently, the intersection of three curves in Hough Space will correspond to the polar coordinates defining the plane spanned by the three points. For practical applications the Hough Space is typically discretized. Each cell in the so-called accumulator corresponds to one plane and a counter is increased for each point that lies on this plane. Among all Hough variants the Randomized Hough Transform (Xu et al., 1990) is the one of most interest to us as Borrmann et al. (2011) concludes that it has "exceptional performance as far as runtime is concerned". Xu et al. (1990) describe the Randomized Hough Transform (RHT) that decreases the number of cells touched by exploiting the fact that a curve with $n$ parameters is defined by $n$ points. For detecting planes, three points from the input space are mapped onto one point in the Hough Space. This point is the one corresponding to the plane spanned by the three points. In each step the procedure randomly picks three points $\mathbf{p}_{1}, \mathbf{p}_{2}$ and $\mathbf{p}_{3}$ from the point cloud. The plane spanned by the three points is calculated as $\rho=\mathbf{n} \cdot \mathbf{p}_{1}=\left(\left(\mathbf{p}_{3}-\mathbf{p}_{2}\right) \times\left(\mathbf{p}_{1}-\mathbf{p}_{2}\right)\right) \cdot \mathbf{p}_{1} \cdot \varphi$ and $\theta$ are
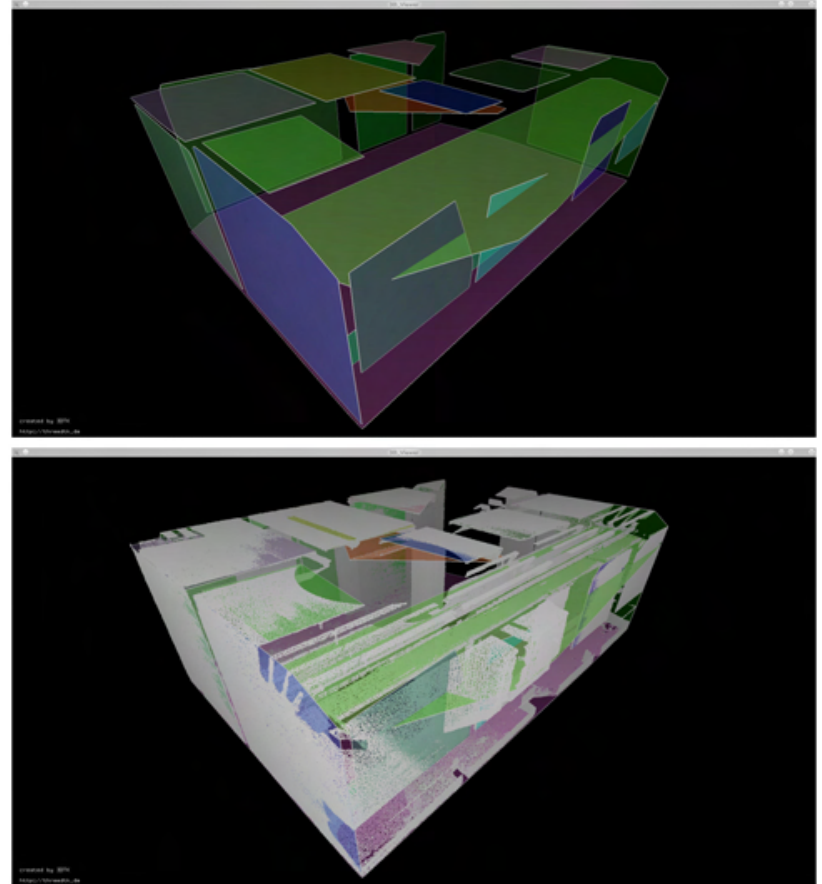

calculated from the normal vectors and the corresponding cell $A(\rho, \varphi, \theta)$ is accumulated. If the point cloud contains a plane with $\rho, \varphi, \theta$, after a certain number of iterations there will be a high score at $A(\rho, \varphi, \theta)$.

When a plane is represented by a large number of points, it is more likely that three points from this plane are randomly selected. Eventually the cells corresponding to actual planes receive more votes and are distinguishable from the other cells. If points are very far apart, they most likely do not belong to one plane. To take care of this and to diminish errors from sensor noise a distance criterion is introduced: $\operatorname{dist} \max \left(\mathbf{p}_{1}, \mathbf{p}_{2}, \mathbf{p}_{3}\right) \leq$ distmax, i.e., the maximum point-to-point distance between $\mathbf{p}_{1}, \mathbf{p}_{2}$ and $\mathbf{p}_{3}$ is below a fixed threshold; for minimum distance, analogous. The basic algorithm is structured as described in Algorithm 1.

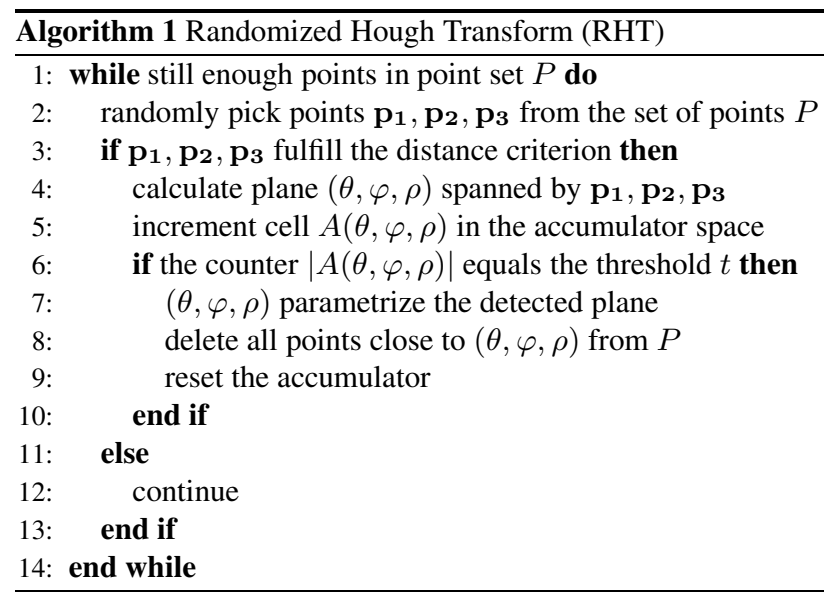

The RHT has several main advantages. Not all points have to be processed, and for those points considered no complete Hough Transform is necessary. Instead, the intersection of three Hough Transform curves is marked in the accumulator to detect the curves one by one. Once there are three points whose plane leads to an accumulation value above a certain threshold $t$, all points lying 
on that plane are removed from the input and hereby the detection efficiency is increased.

Since the algorithm does not calculate the complete Hough Transform for all points, it is likely that not the entire Hough Space needs to be touched. There will be many planes on which no input point lies. This calls for space saving storage procedures that only store the cells actually touched by the Hough Transform. Figure 2 gives an example of the implemented plane detection.

\section{WALL DETECTION}

Starting from the Hough Transform this component returns a collection of planes which are separated into vertical and horizontal surfaces using a predefined epsilon value as part of the initial configuration. All planes that do not fit into this category are simply discarded. We continue with deciding which planes represent the walls, the ceiling and the floor. The ceiling and the floor can easily be extracted from the given information by looking for the lowest and highest horizontal planes. On the other hand, selecting walls from the vertical planes requires additional computation. The implementation of the Hough Transform returns connected planar patches. The center of gravity of the points contained in the hull of a plane corresponds to the application point of the normal in the Hesse normal form. Projecting the points onto a horizontal plane and extracting the concave hull gives a vague notion of the shape of the surface. As opposed to the convex hull the concave hull is not clearly defined. However, its usage enables one to detect the walls of concave rooms.

For each planar patch the concave hull of the points projected onto it is calculated. To determine the actual walls similar planes are grouped and the parameters of all wall candidates representing the same wall are averaged. The resulting wall candidates and the highest and lowest horizontal plane are used to compute the corners of the room by computing the intersection of each wall candidate with the ceiling and the floor. As a result, we now have a set of points representing the corners of the room. Again, we use these points to compute new planar patches, including their
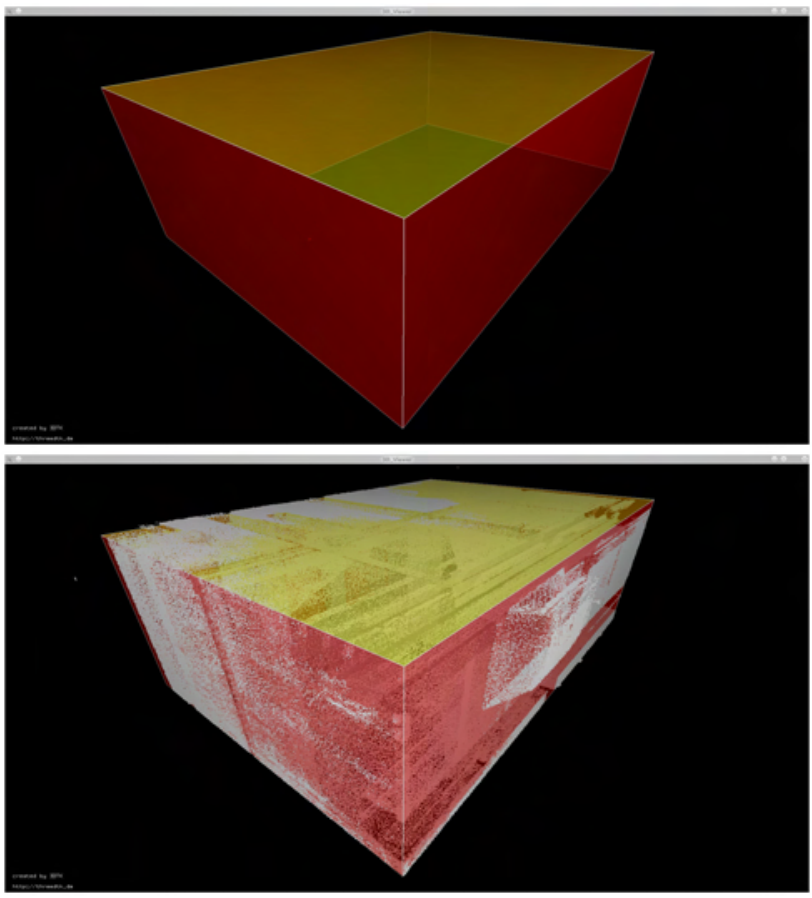

Figure 3: Detected walls in the basement scan. hulls, which are now the final walls, ceiling and floor of the room. See Figure 3 for an example of detected and intersected walls.

\section{OCCLUSION LABELING}

This component of the pipeline is based on approaches borrowed from computer graphics: Octrees (Elseberg et al., 2013), Bresenham's line algorithm and ray tracing (Bresenham, 1965). An octree is a data structure to efficiently store and access spatial point data. The entire space is iteratively divided into voxels of decreasing size. Efficiency is achieved by only further subdividing those voxels that contain data. For occlusion labeling we employ the voxels that intersect with a surface. Each voxel of a surface is assigned one of three possible labels: occupied, empty or occluded. The labeling takes place based on the position of the scanner The decision between empty or occupied is done by checking whether a surface was detected at that location or not, i.e., whether a point was recorded in that area. Distinguishing between an empty or occluded voxel is not possible at this point. This problem is addressed by performing ray tracing to explicitly decide between occluded or empty. Merging data from several scanning positions can help to improve the results by considering the sight of the surface at hand and merging the labels into one unified representation.

This component of the pipeline yields to images for each surface, namely a labels image and a depth image. The labels image will later help us to determine 3 of the 14 features for the Support Vector Machine (SVM) used to classify openings. The depth image will be used to detect lines and construct opening candidates for the SVM. Many features for this component of the pipeline implement ideas from Adan and Huber (2011). The surfaces with openings are of most interest. Figure 4 presents an example of the labels image and the depth image.

\section{OPENING DETECTION}

For this component of our processing pipeline we continue to base our approach on ideas from Adan and Huber (2011). We need to differentiate between actual openings on the surfaces and occluded regions which are basically missing information that needs to be filled in. We make use of the labels and depth images computed in the previous section. A Support Vector Machine (SVM) is used to classify opening candidates and $k$-means clustering is used to determine which candidates belong together.

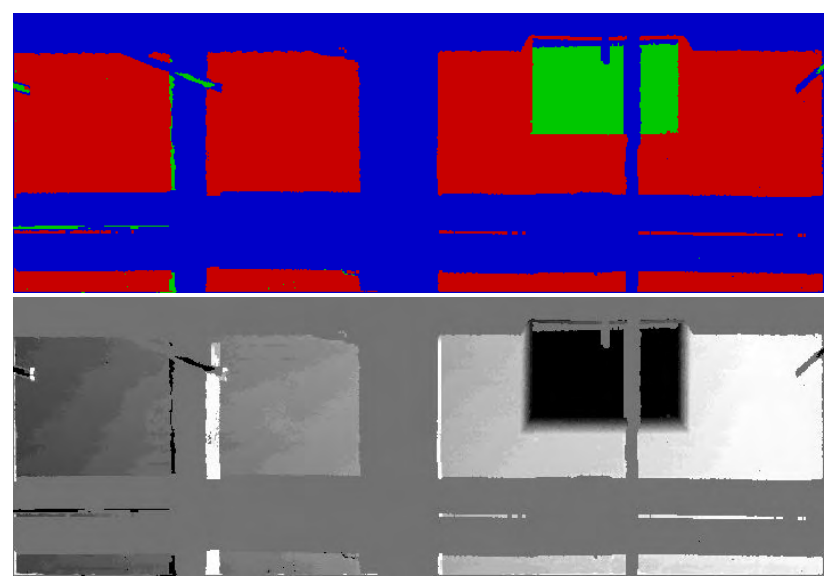

Figure 4: Top: Labels (red occupied, blue occluded, green empty). Bottom: Corresponding depth image. 


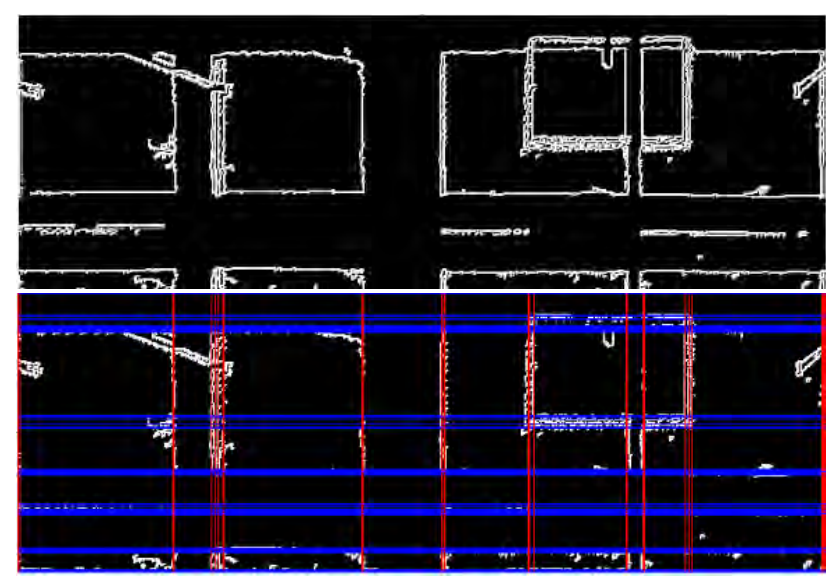

Figure 5: Line detection in generated range images. Top: Image $I_{4}$. Bottom: Hough lines.

The result of this component is a complete $3 \mathrm{D}$ model of the interior and a $2 \mathrm{D}$ mask which is used to reconstruct the point cloud later on.

We start from the depth image $I$ that has been computed previously. We apply the Canny edge detection algorithm and the Sobel Operator on the horizontal and vertical direction. The result is three different images $I_{1}, I_{2}$, and $I_{3}$, respectively. These images are merged into one image and a threshold is applied to every non-zero pixel. This yields a binary image $I_{4}$ with enhanced edges. We then proceed with applying the Hough line detection algorithm on $I_{4}$ which gives us all the lines in the image. We keep only vertical and horizontal lines and construct all the possible rectangles using these lines. Each rectangle represents one opening candidate. Many of these are already discarded taking into consideration various predefined values such as the minimum and maximum area of an opening. The Sobel Operator was used in addition to the Canny edge detector to make the opening detection algorithm more robust, ensuring that it does not miss any important candidates. Hough line detection on Canny edge alone turned out to be not sufficient to detect the proper lines for our data. Figures 5 (top) display the result.

In Figure 5 (bottom) vertical lines are drawn in red and horizontal lines are drawn in blue. We take combinations of two vertical and two horizontal lines to determine an opening candidate. There will be many redundant candidates, where large clusters of horizontal lines have been detected. Once we have determined all the opening candidates, we compute a vector of 14 features for each candidate as described in Adan and Huber (2011): (1) candidate absolute area; (2) candidate width / height ratio; (3) candidate width / surface width ratio; (4) candidate height / surface height ratio; (5) distance from upper side of candidate to upper side of surface; (6) distance from lower side of candidate to lower side of surface; (7) distance from left side of candidate to left side of surface; (8) distance from right side of candidate to right side of surface; (9) root mean squared error to the best plane fitted to the rectangle surface; (10) percentage of occupied area; (11) percentage of occluded area; (12) percentage of empty area; (13) number of interior rectangles; and (14) number of inverted U-shaped rectangles (specific to doors).

The labels image is used to determine features 10,11 and 12 . The feature vectors are used to classify the candidates using a Support Vector Machine (SVM). To train the SVM, a small database was put together with features of actual openings from various buildings across the campus of Jacobs University Bremen, Germany. It is well known that a Support Vector Machine (SVM) is

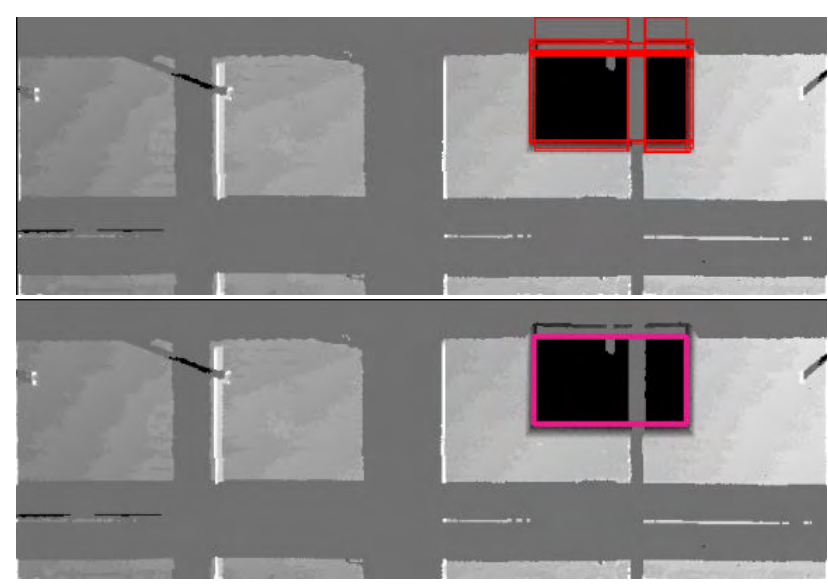

Figure 6: Top: SVM learner applied to the Hough lines as presented in Figure 5. Bottom: Result of $k$-means clustering and check for overlapping candidates.

a learning system that performs classification tasks by constructing hyper planes in a multidimensional space. We used a Radial Basis Function (RBF) as a kernel in a binary SVM model. After training the SVM we give each candidate as input. The SVM decides whether the candidate represents an opening or not. In the end, the candidates left are close representatives of the ground truth used to train the SVM with. Having kept the most relevant opening candidates, many of these actually represent the same opening. We proceed by clustering the candidates using $k$ means clustering. This is a method of cluster analysis that aims to cluster $n$ observations, our opening candidates, into $k$ clusters in which each observation belongs to the cluster with the nearest mean. The features we use to differentiate between the candidates are the center and the absolute area of the candidate, because we might have large candidates from one cluster centered at the same position with smaller candidates. After deciding which cluster the candidates belong to, we need to determine the shape of the final opening. It is important to note that the number of detected clusters is the same with the number of openings on the real surface. We proceed by choosing for each cluster the best candidate which has the largest empty area, and the smallest occupied, and occluded areas. This candidate is the cluster representative and is kept as the final opening that represents that particular cluster (cf. Figure 6).

After computing the final 2D openings on the depth image, we proceed to relabel all remaining empty labels to occluded. This yields the inpaint mask $I_{m}$, that is used for the inpainting (gap filling) algorithm (Salamanca et al., 2008; Adan and Huber, 2011). Basically, the inpaint mask $I_{m}$, tells us what patches of the surface are actually missing information and need to be filled in. Figure 7 (top) shows an inpaint mask. Notice that the amount of reconstruction required for this basement surface is approximately $50 \%$ occluded making it significantly challenging for the reconstruction. In the next component we present a simple way of reconstructing the actual $3 \mathrm{D}$ data from the $2 \mathrm{D}$ depth map and the inpaint mask.

\section{OCCLUSTION RECONSTRUCTION}

The last component of the automated processing is to generate a reconstructed version of the initial point cloud, filling in the missing information induced by occlusions. This component is fairly simple, and requires the use of a gap filling algorithm.

We begin by taking the original depth image $I$, for every surface and apply a gap filling algorithm on it. We used the inpainting al- 

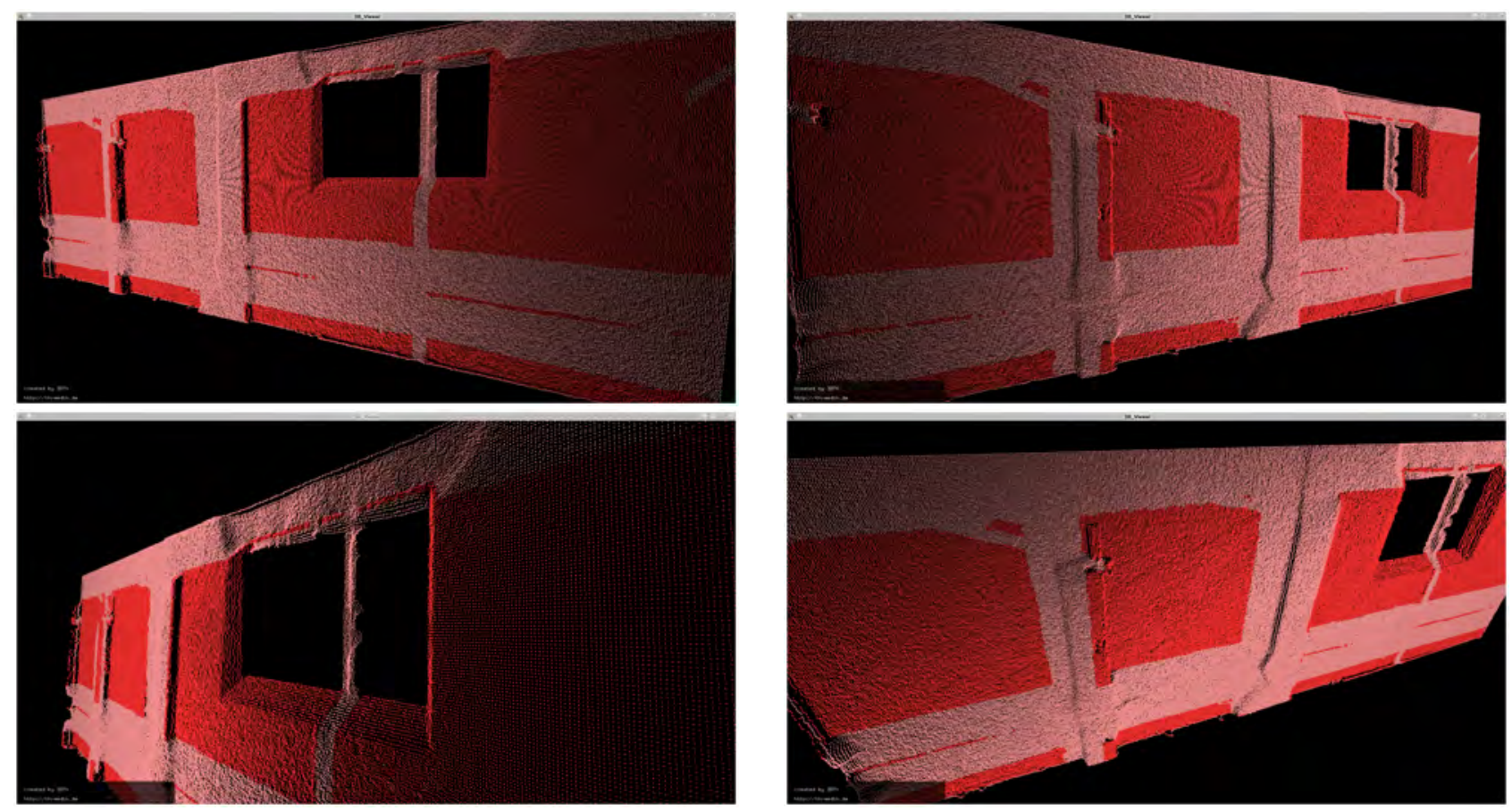

Figure 8: Views of the reconstructed 3D point cloud. The light red points have been added through the inpaint process.

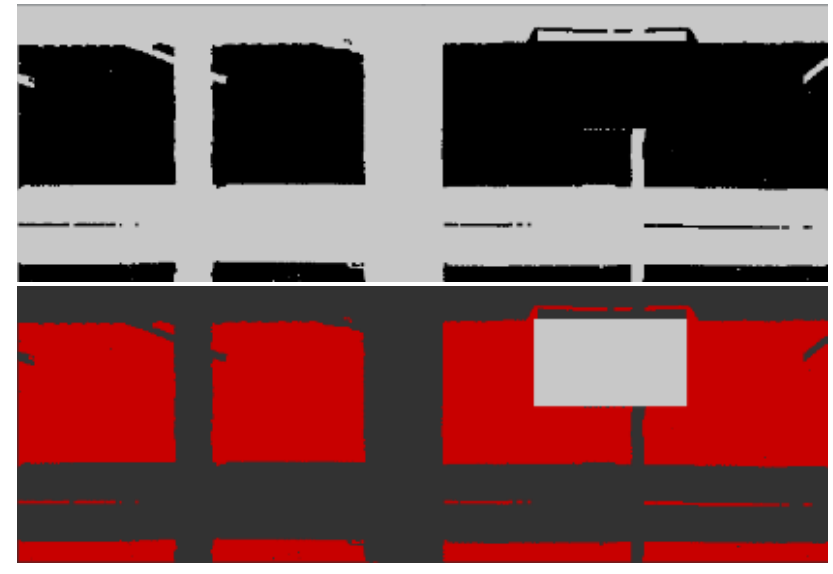

Figure 7: Top: Inpaint mask. Bottom: Final 2D opening.

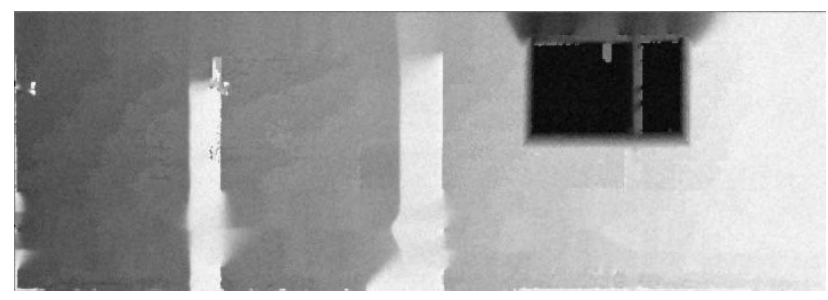

Figure 9: Reconstructed depth image.

gorithm provided in OpenCV, namely the Navier-stokes method (Bertalm et al., 2001). We use the inpaint mask computed in the previous component $I_{m}$. The result of this process is a reconstructed 2D depth image $I_{r}$, having all the missing information filled in. We then proceed to computing the mean and standard deviation of the original depth image, in the depth axis, and add Gaussian noise with the same mean and standard deviation to the newly reconstructed image, thus yielding the final reconstructed depth image of the surface $I_{f}$. Adding Gaussian noise to the reconstructed depth image produces a more natural look and feel of the inpainted parts.
After computing the reconstructed depth image and adding noise, we proceed by projecting $I_{f}$ back into 3D. This yields the reconstructed surface point cloud. Figure 9 shows the reconstructed depth image, from which the 3D point cloud is extrapolated( $\mathrm{cf}$. Figure 8.

\section{EXPERIMENTS AND RESULTS}

The interior modeling software is implemented in $\mathrm{C} / \mathrm{C}++$ and is part of the Open Source software 3DTK - The 3D Toolkit. The input were 3D point clouds acquired by a Riegl VZ-400 terrestrial laser scanner. The point clouds were reduced to approximately 5 million points per 3D scan by applying an octree based reduction down to one point per cubic centimeter.

Throughout the text we have visualized the results of all intermediate steps of our pipeline using a data set acquired in the basement of the Research I building at Jacobs University Bremen, Germany. The basement data is very good for testing our pipeline. Even though it was very fragmented for plane detection, the pipeline was able to accurately determine the six surfaces of

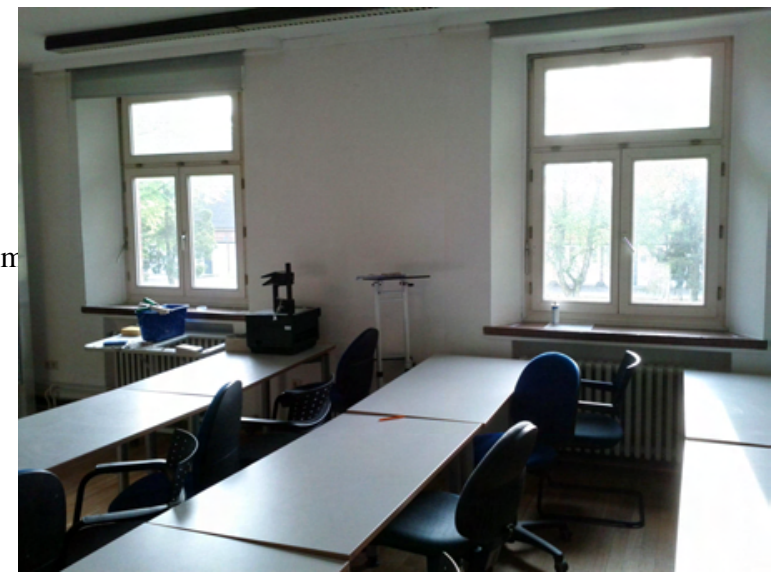

Figure 10: Seminar room. 
the interior. It was ideal for the surface labeling, since it contained large areas of all three labels, mainly occluded areas, due to numerous pipes running from one side of the room to the other. For opening detection, the only opening on the surface is occluded by a vertical pipe, which challenges the SVM in properly choosing candidates. In the case of windows with thick frames or window mounted fans, the algorithm is able to avoid detecting multiple smaller windows, because it checks for overlapping candidates, in the end keeping the best candidate with the largest empty area, which is always the whole window.

Furthermore, we want to report the results on a second data set, which we acquired in a typical seminar room at our university (cf. Figure 10). It features two windows and the walls are less occluded.

For the seminar room data the task for the SVM is simplified since the openings on the surface do not feature any obstructions. Almost all the opening candidates marked in red overlap very closely with the real opening. Clustering is again trivial, as there are clearly two easily distinguishable clusters among the opening candidates. As before, the best candidate from each cluster is chosen as a final opening. We again inpaint the range image and reconstruct the $3 \mathrm{D}$ point cloud as shown in Figure 12.

\section{CONCLUSIONS AND OUTLOOK}

This paper presents a complete and Open Source system for architectural 3D interior reconstruction from 3D point clouds. It consists of fast 3D plane detection, geometry computation by efficiently calculating intersection of merged planes, semantic labeling in terms of occupied, occluded and empty parts, opening detection using machine learning, and finally 3D point cloud inpainting to recover occluded parts. The implementation is based on OpenCV and the $3 D T K$ - The 3D Toolkit and is available on Sourceforge (Lingemann and Nüchter, 2012).

Identifying and restoring hidden areas behind furniture and cluttered objects in indoor scenes is challenging. In this research we approached this task by dividing it into four components, each one relying on the outcome of the previous component. The wall detection and labeling components are highly-reliable, however, for the opening detection and the reconstruction scene specific fine tuning was needed. One reason is that the opening detection vastly depends on the data used to train the Support Vector Machine. This is one of the drawbacks when using supervised learning methods. Moreover, we considered windows to be of rectangular shape. Although, this is a valid assumption, attempting to detect windows of various shapes would severely increase the difficulty of the task. For the reconstruction component, despite the fact that we are being provided a reliable inpaint mask from the previous component, it is difficult to assume what is actually in the occluded areas. 2D inpainting is one approach in approximating what the surface would look like. We have to state, that the best solution to reducing occlusion is to take multiple scans. Thus, future work will incorporate multiple scans and address the next-best-view planning problem.

So far we have presented a pipeline based on four major components. This modularity offers many advantages as each component is replaceable by any other approach. In the future, other components will be added and we will allow for any combination of these components to be used. One potential improvement for the opening detection component would be the use of reflectance images. This would greatly increase the robustness of this component. We would be able to generate fewer, more appropriate opening candidates, instead of generating all the possible ones from the extracted lines. This can be achieved by exploiting the intensity information contained in the reflectance image.

Needless to say a lot of work remains to be done. The current purpose of our software is to simplify and automate the generation of building models. Our next step will be to extend the pipeline with an additional component that will put together multiple scans of interiors and generate the model and reconstructed point cloud of an entire building. Another way of extending the pipeline is to add a component that would combine geometrical analysis of point clouds and semantic rules to detect 3D building objects. An approach would be to apply a classification of related knowledge as definition, and to use partial knowledge and ambiguous knowledge to facilitate the understanding and the design of a building (Duan et al., 2010).

\section{References}

Adan, A. and Huber, D., 2011. 3D reconstruction of interior wall surfaces under occlusion and clutter. In: Proceedings of 3D Imaging, Modeling, Processing, Visualization and Transmission (3DIMPVT).

Adan, A., Xiong, X., Akinci, B. and Huber, D., 2011. Automatic creation of semantically rich 3D building models from laser scanner data. In: Proceedings of the International Symposium on Automation and Robotics in Construction (ISARC).

Bertalmio, M., Bertozzi, A. and Sapiro, G., 2001. Navier-stokes, fluid dynamics, and image and video inpainting. In: Proceedings of the IEEE Conference on Computer Vision and Pattern Recognition (CVPR 01), pp. $355-362$

Böhm, J., 2008. Facade detail from incomplete range data. In: Proceedings of the ISPRS Congress, Beijing, China.

Böhm, J., Becker, S. and Haala, N., 2007. Model refinement by integrated processing of laser scanning and photogrammetry. In: Proceedings of 3D Virtual Reconstruction and Visualization of Complex Architectures (3DArch), Zurich, Switzerland.

Borrmann, D., Elseberg, J., Lingemann, K. and Nüchter, A., 2011. The 3D Hough Transform for plane detection in point clouds: A review and a new accumulator design. 3D Research.

Bresenham, J. E., 1965. Algorithm for computer control of a digital plotter. IBM Systems Journal 4 4(1), pp. 25-30.

Budroni, A. and Böhm, J., 2005. Toward automatic reconstruction of interiors from laser data. In: Proceedings of 3D-Arch, Zurich, Switzerland

Duan, Y., Cruz, C. and Nicolle, C., 2010. Architectural reconstruction of 3D building objects through semantic knowledge management. In: ACIS International Conference on Software Engineering, Artificial Intelligence, Networking and Parallel/Distributed Computing.

Elseberg, J., Borrmann, D. and Nüchter, A., 2013. One billion points in the cloud - an octree for efficient processing of $3 \mathrm{~d}$ laser scans. Journal Potogrammetry and Remote Sensing.

Fisher, R. B., 2002. Applying knowledge to reverse engeniering problems. In: Proceedings of the International Conference. Geometric Modeling and Processing (GMP '02), Riken, Japan, pp. 149 - 155.

Früh, C., Jain, S. and Zakhor, A., 2005. Data processing algorithms for generating textured 3D building facade meshes from laser scans and camera images. International Journal of Computer Vision (IJCV) 61(2), pp. 159-184.

Hähnel, D., Burgard, W. and Thrun, S., 2003. Learning compact 3D models of indoor and outdoor environments with a mobile robot. Robotics and Autonomous Systems 44(1), pp. 15-27.

Hough, P., 1962. Method and means for recognizing complex patterns. US Patent 3069654

Lingemann, K. and Nüchter, A., 2012. The 3D Toolkit. http://threedtk.de. 

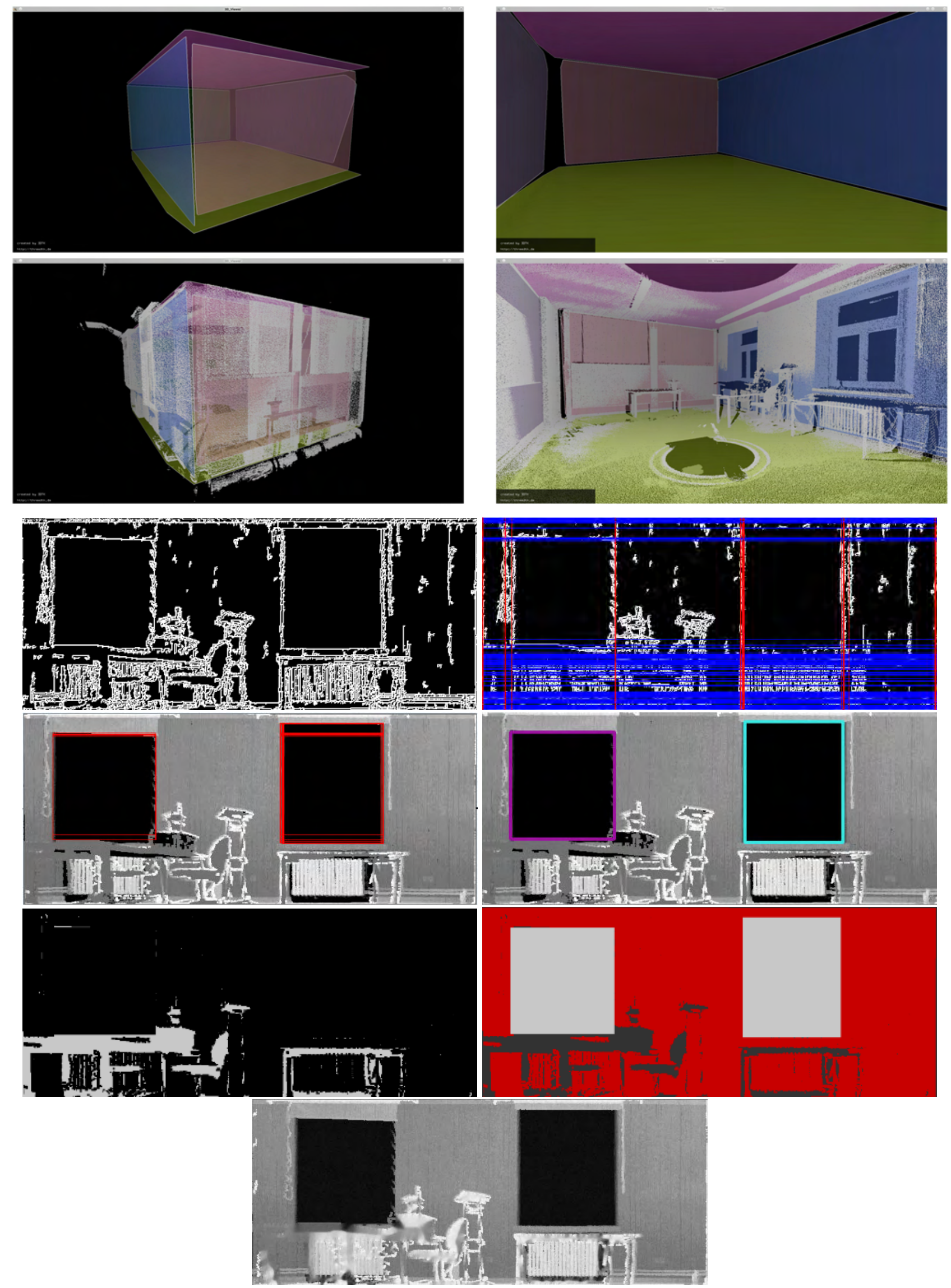

Figure 11: Top row: Detected planes in a seminar room. Second row: Detected planes as overlay to the 3D point cloud. Third row: Image $I_{4}$ and the Hough lines. Forth row: Opening candidates and the result of the $k$-means clustering. Fifth row: Inpaint mask and final 2D openings. Bottom: Reconstructed depth image. 

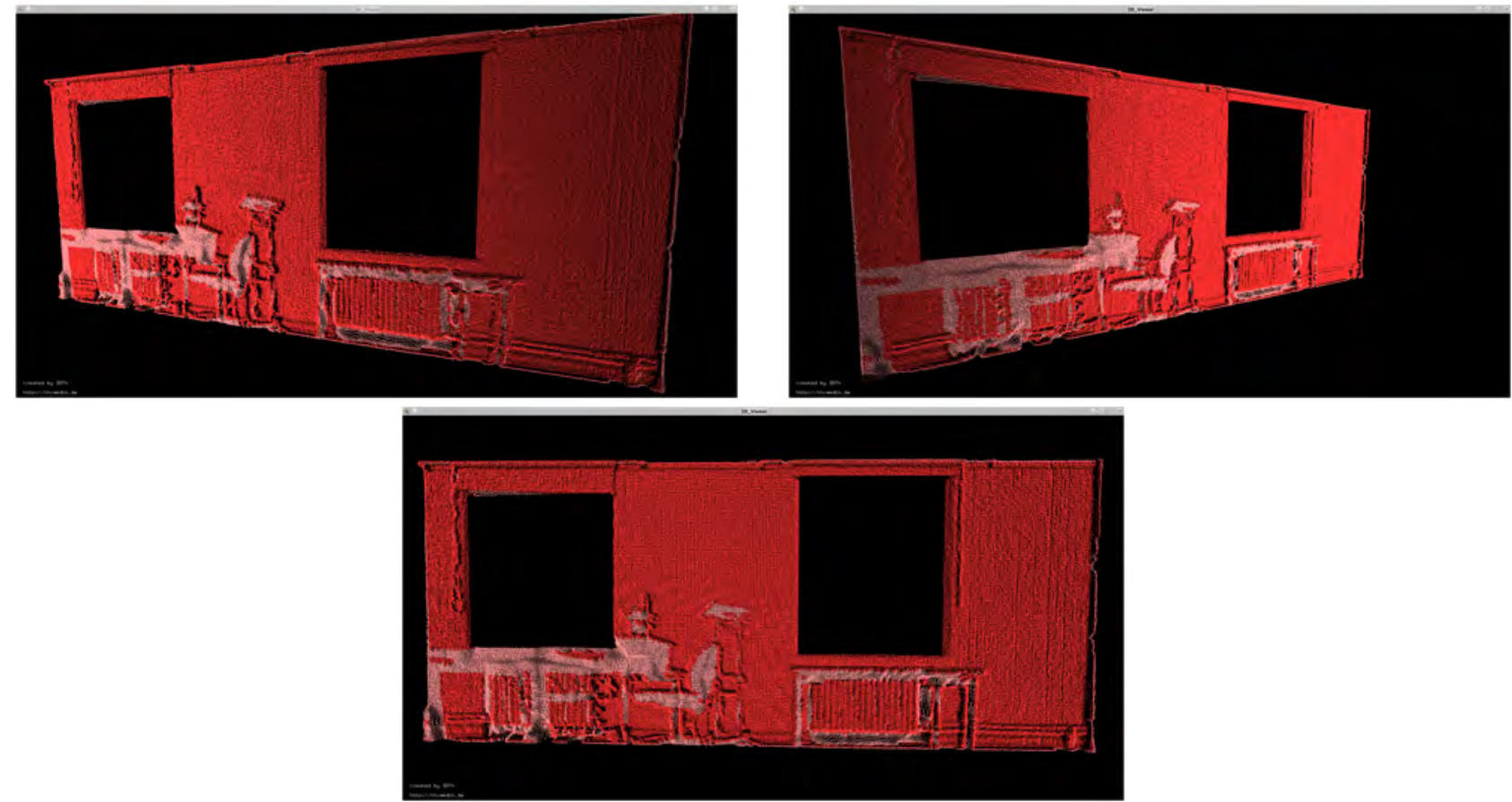

Figure 12: Reconstructed 3D point clouds in the seminar room.

Nüchter, A., Surmann, H., Lingemann, K. and Hertzberg, J., 2003. Semantic Scene Analysis of Scanned 3D Indoor Environments. In: Proceedings of the of the 8th International Fall Workshop Vision, Modeling, and Visualization (VMV '03), Munich, Germany, pp. 215-222.

$\mathrm{Pu}, \mathrm{S}$. and Vosselman, G., 2009. Knowledge based reconstruction of building models from terrestrial laser scanning data. Journal of Photogrammetry and Remote Sensing 64(6), pp. 575-584.

Ripperda, N. and Brenner, C., 2009. Application of a formal grammar to facade reconstruction in semiautomatic and automatic environments. In: Proceedings of AGILE Conference on Geographic Information Science, Hannover, Germany.

Salamanca, S., Merchan, P., Perez, E., Adan, A. and Cerrada, C., 2008. Filling holes in 3D meshes using image restoration algorithms. In: Proceedings of the Symposium on 3D Data Processing, Visualization, and Transmission (3DPVT), Atlanta, Georgia, United States of America.
Stamos, I., Yu, G., Wolberg, G. and Zokai, S., 2006. Application of a formal grammar to facade reconstruction in semiautomatic and automatic environments. In: Proceedings of 3D Data Processing, Visualization, and Transmission (3DPVT), pp. 599-606.

Thrun, S., Martin, C., Liu, Y., Hähnel, D., Montemerlo, R. E., Chakrabarti, D. and Burgard, W., 2004. A real-time expectation maximization algorithm for acquiring multi-planar maps of indoor environments with mobile robots. IEEE Transactions on Robotics 20(3), pp. 433 443.

Willow Garage, 2012. Point cloud library. http://pointclouds.org/.

Xu, L., Oja, E. and Kultanen, P., 1990. A new curve detectionmethod: Randomized Hough Transform (RHT). Pattern Recognition Letters 11, pp. 331-338. 